\title{
Papers
}

\section{Systematic review of topical treatments for fungal infections of the skin and nails of the feet}

\author{
Rachel Hart, Sally E M Bell-Syer, Fay Crawford, David J Torgerson, Philip Young, Ian Russell
}

\begin{abstract}
Objective To identify and synthesise the evidence for efficacy and cost effectiveness of topical treatments for superficial fungal infections of the skin and nails of the feet.

Design Systematic review.

Interventions Topical treatments for superficial fungal infections.

Main outcome measures Cure confirmed by culture and microscopy for skin and by culture for nails in patients with clinically diagnosed fungal infections. Results Of 126 trials identified in 121 papers, 72 $(57.1 \%)$ met the inclusion criteria. Placebo controlled trials yielded pooled relative risks of failure to cure skin infections: allylamines $(0.30,95 \%$ confidence interval 0.24 to 0.38$)$; azoles $(0.54,0.42$ to 0.68$)$; undecenoic acid $(0.28,0.11$ to 0.74$)$; and tolnaftate $(0.46,0.17$ to 1.22$)$. Although meta-analysis of 11 trials comparing allylamines and azoles showed a relative risk of failure to cure of 0.88 (0.78 to 0.99$)$ in favour of allylamines, there was evidence of language bias. Seven reports in English favoured allylamines (0.79, 0.69 to 0.91 ), but four reports in foreign languages showed no difference between the two drugs (1.01, 0.90 to 1.13$)$. Neither trial of nail infections showed significant differences between alternative topical treatments.

Conclusions Allylamines, azoles, and undecenoic acid were efficacious in placebo controlled trials. There are sufficient comparative trials to judge relative efficacy only between allylamines and azoles. Allylamines cure slightly more infections than azoles but are much more expensive than azoles. The most cost effective strategy is first to treat with azoles or undecenoic acid and to use allylamines only if that fails.
\end{abstract}

\section{Introduction}

About $15 \%$ of the population of the United Kingdom have fungal infections of the foot. ${ }^{1}$ The main treatments are topical fungistatic or fungicidal preparations, some of which are available over the counter. We report a systematic review of randomised controlled trials of topical antifungal treatments for dermatophyte infections of the skin and nails of the foot, which were designed to assess efficacy and cost effectiveness.

\begin{abstract}
Methods
Search strategy

We searched Medline, Embase, CINAHL, BIDS, the Cochrane Controlled Trials Register, CAB-Health, Healthstar, DARE, the NHS Economic Evaluation Database, and Econlit to December 1997. We searched by hand Foot, the Journal of British Podiatric Medicine, and the British Journal of Podiatric Medicine and Surgery; the last two are not listed in any databases. We obtained the details of the Cochrane Skin Group's recent search by hand of the British Journal of Dermatology. We searched the bibliographies of all review papers identified. To identify unpublished or unlisted trials, we contacted international pharmaceutical companies and all schools of podiatry in the United Kingdom.
\end{abstract}

\section{Selection criteria}

We considered all randomised controlled trials that evaluated topical treatments for fungal infections of the skin and nails of the foot. For skin infections we included only trials that used microscopy and culture to establish the presence of dermatophytes. For nail infections we included only trials that used culture to establish the presence of dermatophytes. We included duplicate trials only once. We excluded trials covering sites other than the foot where data related specifically to the foot could not be extracted. Two reviewers (RH and SEMBS) independently applied these criteria to each trial located. No language restrictions applied.

\section{Data extraction and quality assessment}

Both reviewers independently summarised the trials included, and they appraised their quality of reporting using a structured data extraction tool of 12 quality criteria. The criteria were: aims clearly defined; prior sample size calculation reported; inclusion and exclusion criteria defined; subjects blinded; method of randomisation defined; baseline comparability of groups reported (age, sex, and duration of complaint); interventions defined; outcome assessment blinded; compliance assessed; and trial analysed by intention to treat. $^{2-4}$

\section{Statistical analysis}

For each trial we calculated the cure rates at follow up from the reported mycological results-that is, negative results on microscopy, and no growth of dermatophyte in culture. We then estimated the difference, with 95\%

\author{
Editorial by Finlay \\ Faculty of \\ Community Health \\ Sciences, University \\ of Wales Institute \\ Cardiff, Cardiff \\ CF5 2SG \\ Rachel Hart, \\ research podiatrist \\ Department of \\ Health Sciences \\ and Clinical \\ Evaluation, \\ University of York, \\ YO10 5DD \\ Sally E M Bell-Syer, \\ research fellow \\ Fay Crawford, \\ Medical Research \\ Council fellow \\ Philip Young, \\ lecturer in biostatistics \\ Ian Russell, \\ professor of health \\ sciences \\ Centre for Health \\ Economics, \\ University of York \\ David J Torgerson, \\ senior research fellow \\ Correspondence to: \\ F Crawford \\ fc5@york.ac.uk \\ BMJ 1999;319:79-82
}

website

extra

Details of the trials appear on the BMJ's website

www.bmj.com 
confidence intervals, in the proportion of patients cured. To estimate differences between treatments we pooled trials that evaluated similar interventions and controls. As there was clear evidence of heterogeneity between trials $(\mathrm{P}<0.001)$, we used random effects models. We calculated the relative risk of failure to cure, with $95 \%$ confidence intervals. ${ }^{5}$

\section{Economic analysis}

We calculated cost effectiveness in four ways: cost per cure of using an over the counter preparation; marginal cost (including general practitioner's time) per cure of a podiatrist referring a patient to a general practitioner for treatment with an allylamine rather than providing an over the counter preparation; marginal cost per cure (excluding general practitioner's time) of a general practitioner, once consulted, prescribing an allylamine rather than an over the counter preparation; and marginal cost per cure (excluding general practitioner's time) of a general practitioner, once consulted, treating first with an azole and reserving allylamines for treatment failures.

\section{Results}

\section{Identified trials of skin}

We located 111 papers reporting trials of topical treatments for fungal skin infections, and we included $65(58.6 \%)$ of these in our review..$^{6-10}$ w1-w60 As one of these papers ${ }^{8}$ reported three distinct trials, and three papers $^{7} \mathrm{w}^{\mathrm{w} 3}$ each reported a pair of distinct trials, we included 70 trials in all. Thirty one trials in 29 papers $^{6-10}$ w1-w24 $^{-1}$ compared a single active treatment with placebo (see website). Twenty seven trials in 26 papers $^{8}{ }^{\text {w2 } 2}$ w25-w48 compared two active treatments (see website). Twelve trials ${ }^{\mathrm{w} 49-\mathrm{w} 60}$ compared more than two treatments within the same trial (see website). We excluded the remaining 46 trials ${ }^{\text {w61-w106 }}$ owing to duplicate reporting, combining data from more than one anatomical site (for example, hand and foot), or absence of mycological testing. Responses from the authors of provisionally excluded trials enabled us to include just one. ${ }^{\mathrm{w} 29}$ Responses from the pharmaceutical industry identified no additional studies.

\section{Identified trials of nails}

Apart from one trial (already excluded) of topical treatments for skin and nail infections, ${ }^{\text {w99 }}$ we found seven trials evaluating the efficacy of topical treatments for nails, and we included two of these. ${ }^{\text {w107 w108 }}$ The other five trials reported combined data from fingernails and toenails. ${ }^{\text {w109-w133 }}$ We also excluded three trials evaluating a combination of systemic and topical treatments for infected nails. ${ }^{\mathrm{w} 114-\mathrm{w} 116}$

\section{Quality assessment}

The mean number of quality criteria met by the 72 included trials (two for nail infections) was only 6.3 out of 12. Only 19 trials reported the method of randomi-

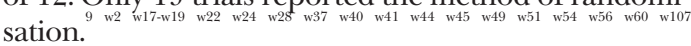
Blinded outcome assessment was reported in only 10 trials. $^{\text {w8 }}$ w18 w22 w30 w31 w37 w41 w49 w54 w61 Only seven trials, however, did not report blinding of participants. $^{8 \text { w17 w25 w26 w32 w47 w48 }}$

\section{Included trials of skin}

The 70 trials evaluated a variety of treatments. Azoles were assessed in 46 trials, allylamines in 27 , tolnaftate in five, ${ }^{\mathrm{w} 30 \mathrm{w} 44 \mathrm{w} 56-\mathrm{w} 58}$ and undecenoic acid in four. ${ }^{\mathrm{w} 24 \text { w56 w57 w60 }}$ Ciclopiroxolamine, which is available over the counter in the United States and parts of Europe but not in the United Kingdom, was evaluated in two trials reported in the same paper. ${ }^{\mathrm{w} 2}$ Griseofulvin, ${ }^{10}$ haloprogen, ${ }^{\text {w30 }}$ tea tree oil, ${ }^{\mathrm{w} 58}$ and tolciclate ${ }^{\mathrm{w} 21}$ were each evaluated in a separate small trial. Variotin and Whitfield's ointment were compared with each other in one very small trial, ${ }^{\mathrm{w} 41}$ which followed patients for 24 weeks. As no other trial followed patients for more than 12 weeks, no conclusions are possible about differences in relapse rates.

All included studies reported the infecting species identified from the initial culture. Except in one trial ${ }^{\mathrm{w} 27}$ all fungal organisms present were dermatophytes, most commonly Trichophytum rubrum. Although our review was restricted to trials reporting mycological cure, no trial reported the species obtained from patients who were not cured.

\section{Azoles versus placebo}

Meta-analysis of data from 17 trials comparing azoles with placebos ${ }^{6-9}$ w1 w12-w20 w55 w59 estimated the pooled relative risk of failure to cure as 0.54 (95\% confidence interval 0.42 to 0.68 ). The concentration of these drugs was generally $1 \%$ but $2 \%$ for miconazole.

\section{Allylamines versus placebo}

Meta-analysis of data from 12 trials comparing allylamines (concentration of $1 \%$ in all) with placebos estimated the relative risk of failure to cure as 0.30 (0.24 to 0.38$)$.

\section{Azoles versus allylamines}

The efficacy of azoles seems to depend on the duration of treatment. Ablon et al reported that 2 weeks of treatment with oxiconazole cured only $27 \%$ of patients. ${ }^{\text {w4 }}$ More rigorously Bergstresser et al reported a randomised comparison showing that 1 week of treatment with clotrimazole cured only $35 \%$ of patients whereas 4 weeks of treatment cured 70\%. ${ }^{\text {w53.3 }}$ Therefore we excluded both Ablon's study and Bergstresser et al's group treated with azoles for only 1 week from our meta-analysis of azoles versus allylamines. This left 12 trials, ${ }^{\mathrm{w} 27}$ w29 w31-w33 w36-w40 w50 w53 which included three azoles (bifonazole, clotrimazole, and miconazole) and two allylamines (naftifine and terbinafine). Where stated the concentration was $1 \%$ for all agents. The frequency of treatment was once or twice daily, generally for 4 or more weeks. The pooled relative risk of failure to cure of 0.88 ( 0.78 to 0.99 ) favoured allylamines and was just significant (figure). There was, however, a statistically significant difference between the relative risk estimates from papers in English and other languages. Eight English language reports ${ }^{\mathrm{w} 29}$ w31 w36 w37 w39 w40 w50 w53 favoured allylamines $(0.79,0.69$ to 0.91$)$, but four foreign language reports ${ }^{\text {w27 }}$ w32 w33 w38 showed no difference between the two drugs (1.01, 0.90 to 1.13$)$.

\section{Other compounds}

The three placebo controlled trials of $1 \%$ tolnaftate $\mathrm{w}^{\mathrm{w} 56-\mathrm{w} 58}$ yielded a pooled relative risk of failure to cure of 0.46 (0.17 to 1.22). The two small comparative trials showed 


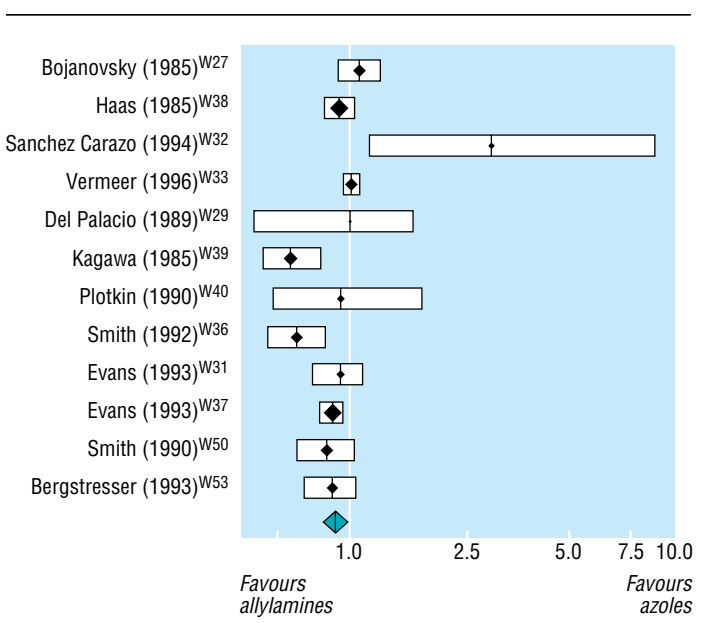

Comparative trials of topical allylamines and topical azoles for fungal skin infections of foot: relative risk plot summarised by random effects model ${ }^{5}$

tolnaftate to be significantly worse than haloprogen ${ }^{\text {w30 }}$ and profoundly, though not significantly, worse than clotrimazole. ${ }^{\mathrm{w}+3}$ The four placebo controlled trials of $5 \%$ undecenoic acid-three with just two groups ${ }^{\mathrm{w} 24} \mathrm{w56}$ w57 and one that also compared undecenoic acid with zinc undecenoate ${ }^{\mathrm{w} 60}$-yielded a pooled relative risk of failure to cure of 0.28 (0.11 to 0.74$)$. Ciclopiroxolamine was significantly better than placebo $(0.14,0.06$ to 0.32$)$ and better than clotrimazole although not significantly $(0.89,0.72$ to 1.10$) .^{\text {w2 }}$

\section{Included trials of nails}

In the smaller trial, two amorolfine $5 \%$ nail lacquer formulations with different vehicles both achieved a cure rate close to $90 \%$ after 6 weeks. ${ }^{\text {w108 }}$ In the larger trial, clotrimazole solution and tea tree oil both achieved a cure rate close to $10 \%$ after 6 months. ${ }^{\text {w107 }}$

\section{Costs and cost effectiveness}

The table shows differences in cure rates and costs for the main treatments and their cost effectiveness. So few trials compared azoles, undecenoic acid, and tolnaftate directly that confidence intervals for their costs per cure must be on the basis of placebo controlled trials. Although there are only four such trials of undecenoic acid, their findings are much more homogeneous than trials of other drugs. Undecenoic acid therefore yields a significantly cheaper average cost per cure when purchased over the counter than azoles, and a much narrower confidence interval than tolnaftate. As azoles cost the NHS about half the retail price, however, there is no significant difference in NHS cost per cure between azoles and undecenoic acid, regardless of general practitioner's time.

The NHS cost of allylamines is about 2.5 times that of over the counter preparations. Although allylamines are more efficacious than azoles, the estimated difference in cure rate is small. Marginal analysis in the table shows that the extra cost of the allylamines needed to achieve this gain in efficacy is about five times the marginal cost of the azoles. Thus one should consider using an azole first, costing $£ 195$ for a cohort of 100 patients, and an allylamine only if that fails. Pooled analysis of the trials directly comparing azoles with allylamines yielded estimated cure rates of $72 \%$ and $80 \%$ respectively. If the expected 28 patients not cured by azoles were then treated with an allylamine, this would cost a further $£ 136$. The total cost would be $£ 331$ ( $£ 4.14$ for each of the 80 cured patients) rather than the $£ 486$ ( $£ 6.08$ per cured patient) needed to treat all patients with an initial allylamine.

\section{Discussion}

Athlete's foot is widespread and infectious. Initial treatment for most patients is a topical cream or ointment. Good evidence shows that allylamines, azoles, and undecenoic acid are efficacious compared with placebo. Unfortunately there is little evidence to assess tolnaftate against placebo or to compare azoles, undecenoic acid, and tolnaftate with each other.

More trials compared allylamines with azoles. The resulting meta-analysis suggests that allylamines are generally more efficacious, with a relative risk of failure

Cost effectiveness of alternative topical treatments for fungal skin infections of foot. Costs are in $£$

\begin{tabular}{|c|c|c|c|c|c|c|c|}
\hline \multirow[b]{2}{*}{$\begin{array}{l}\text { Comparison } \\
\text { (No of trials) }\end{array}$} & \multirow[b]{2}{*}{$\begin{array}{l}\% \text { Pooled estimate of } \\
\text { difference in cure } \\
\text { rate }(95 \% \mathrm{CI})\end{array}$} & \multirow[b]{2}{*}{ Retail price } & \multicolumn{2}{|c|}{ NHS cost } & \multirow[b]{2}{*}{$\begin{array}{l}\text { OTC cost per cure } \\
(95 \% \mathrm{Cl})\end{array}$} & \multicolumn{2}{|c|}{ Marginal NHS cost } \\
\hline & & & $\begin{array}{l}\text { Excluding GP time } \\
\text { (unless stated) }\end{array}$ & Including GP time & & $\begin{array}{l}\text { Per cure excluding GP } \\
\text { time }(95 \% \mathrm{Cl})\end{array}$ & $\begin{array}{l}\text { Per cure including GP } \\
\text { time }(95 \% \mathrm{CI})\end{array}$ \\
\hline $\begin{array}{l}\text { Azoles } v \text { placebo } \\
\text { (17 trials) }\end{array}$ & 37.6 (25.3 to 49.9$)$ & 3.89 & 1.95 & $1.95+9.00=10.95$ & 10.34 (7.80 to 15.38$)$ & 5.19 (3.91 to 7.71$)$ & 29.12 (21.94 to 43.28$)$ \\
\hline $\begin{array}{l}\text { Allylamines } v \text { azoles } \\
\text { (12 trials) }\end{array}$ & $7.9(0.01$ to 16.3$)$ & & $\begin{array}{l}\text { If patient is } \\
\text { consulting GP: } \\
2.91-0.071^{\star} \times \\
9.00 \dagger=2.27 \\
\text { If patient is } \\
\text { consulting podiatrist: } \\
11.91-0.071^{\star} \times \\
9.00 \dagger=11.27\end{array}$ & & Not OTC & $\begin{array}{l}28.73 \ddagger \text { (lower bound } \\
\text { 13.92; no upper bound) }\end{array}$ & $\begin{array}{c}143 \S \text { (lower bound } \\
69.14 ; \text { no upper bound) }\end{array}$ \\
\hline $\begin{array}{l}\text { Undecenoic acid } v \\
\text { placebo ( } 4 \text { trials) }\end{array}$ & 46.1 (31.6 to 60.6$)$ & 1.89 & 1.89 & $1.89+9.00=10.89$ & 4.10 (3.12 to 5.98$)$ & 4.10 (3.12 to 5.98$)$ & 23.62 (17.97 to 34.46$)$ \\
\hline $\begin{array}{l}\text { Tolnaftate } v \text { placebo } \\
\quad(3 \text { trials })\end{array}$ & 37.6 (2.6 to 72.5$)$ & 1.83 & 1.83 & $1.83+9.00=10.83$ & 4.87 (2.52 to 69.58$)$ & 4.87 (2.52 to 69.58$)$ & 28.80 (14.94 to 411$)$ \\
\hline
\end{tabular}

OTC=over the counter; $\mathrm{GP}=$ general practitioner

${ }^{*}$ Since allylamines cure $7.9 \%$ more infections than azoles on average, it is assumed that initial treatment with them will reduce consultations with GP by $7.9 \%$.

†Average cost of consultation with GP in his or her surgery is $£ 9.00$. w118

¥When GP is considering during consultation initiated by patient or podiatrist whether to prescribe terbinafine or clotrimazole as first treatment.

§When podiatrist is considering referring patient to GP for treatment with terbinafine rather than providing clotrimazole. 
We thank Kath Cross, Zelda Di Blasi, Alison Eastwood, Annelise Emmans, Jill Ferrari, Nick Freemantle, Simon Gilbody, Mark Goodfield, Rod Hay, Mark Petticrew, Daphne Russell, Trevor Sheldon, Fujian Song, Wendy Tyrrell, Hywel Williams and the Cochrane Skin Group, and the referees acting for the Wales Office of Research and Development for Health and Social Care.

Contributors: RH and SEMB-S contributed to the development of the protocol (including search strategy and data extraction form), identified and selected trials, extracted and tabulated data, contributed to their analysis, and reviewed the paper. FC initiated and managed the review, led the development of the protocol, arbitrated in the selection of trials, contributed to the analysis and interpretation of data, and wrote all drafts of the paper but the last. DJT helped to initiate the review, contributed to the statistical analysis and interpretation of data, undertook and wrote up the economic analysis, and reviewed the paper. PY undertook and wrote up the statistical analysis, contributed to the interpretation of data, and reviewed the paper. IR helped to initiate the review, contributed to its management, and wrote the final draft of the paper. FC and IR will act as guarantors for the paper.

Funding: Wales Office of Research and Development for Health and Social Care.

Competing interests: None declared.

to cure of 0.88 ( 0.78 to 0.99$)$. This difference is more marked in trials reported in English $(0.79,0.69$ to 0.91$)$ but not significant in foreign language trials. This discrepancy could not be explained by differences in the quality of the trials, commercial sponsorship, or the patients' characteristics. This suggests that by including foreign papers in this review we have reduced English language bias ${ }^{\text {w119 }}$ and improved the estimate of relative risk of failure to cure.

We detected no differences in efficacy between individual allylamines or individual azoles. As no trial reported the species obtained from patients who were not cured, we cannot draw conclusions about susceptibility to individual compounds to help clinical decision making. Without more evidence we recommend treatment with the least expensive compound within each class.

Although allylamines cure slightly more infections than azoles, they are available only on prescription at much greater cost. Hence the most cost effective strategy is to treat first with an azole or undecenoic acid and to reserve allylamines for treatment failures. To improve on this recommendation more direct comparisons are needed of undecenoic acid and tolnaftate with allylamines and azoles, and a large trial is needed to refine our estimate of the incremental benefit of allylamines over azoles.

Evidence about the efficacy of topical treatments for nail infections is very sparse. Little can be concluded about the role of these agents in curing infected toenails. Rigorous research is overdue.

\section{Conclusion}

In summary there is little evidence to differentiate between three popular over the counter topical treatments for fungal skin infections. Azoles may be more efficacious than tolnaftate. They seem, however, no more efficacious than undecenoic acid. Evidence shows that allylamines are slightly more efficacious than azoles and other over the counter treatments but at much greater cost. Thus we recommend initial treatment with azoles or undecenoic acid, and the use of allylamines only if that fails.

1 Gentles JC, Evans EGV. Foot infections in swimming baths. BMJ 1973;3:260-2.

2 Moher D, Jadad AR, Nichol G, Penman M, Tugwell P, Walsh S. Assessing the quality of randomised controlled trials: an annotated bibliography of scales and checklists. Control Clin Trials 1995;16:62-73.

3 Jadad AR, Moore RA, Carroll D, Jenkinson C, Reynolds DJM, Gavaghan DJ, et al. Assessing the quality of reports of randomised clinical trials: is blinding necessary? Control Clin Trials 1996;17:1-12

4 Begg C, Cho M, Eastwood S, Horton R, Moher D, Olkin I, et al. Improving the quality of randomised controlled trials: the CONSORT statement. JAMA 1996;276:637-9.

5 DerSimonian R, Laird N. Meta-analysis in clinical trials. Control Clin Trials 1986;7:177-88.

6 Smith EB, Graham JL, Ulrich JA. Topical clotrimazole in tinea pedis. South Med J 1977;70:47-8.

7 Speikerman PH, Young MD. Clinical evaluation of clotrimazole-a broad-spectrum antifungal agent. Arch Dermatol 1976;112:350-2.

8 Smith EB, Becker LE, Tschen EH, Way B, Way A. Topical tioconazole in tinea pedis. Adv Ther 1988;5:313-8.

9 Akers WA, Lane A, Lynfield Y, Greenberg J, Hall J, Mangan C, et al. Sulconazole nitrate $1 \%$ cream in the treatment of chronic moccasin-type tinea pedis caused by trichophyton rubrum. J Am Acad Dermatol 1989;21:686-9.

10 Aly R, Bayles CI, Oakes RA, Bibel DJ, Maibach HI. Topical griseofulvin in the treatment of dermatophytoses. Clin Exp Dermatol 1994;19:43-6.

(Accepted 28 April 1999)

\section{Endpiece \\ Do texts in English encourage quacks?}

The introduction to Theophilus Lobb's Treatise on the Small Pox (T Woodward, London 1741) has the following passage:

"Some Persons have objected that Writing physical Books in English is the Way to make QUACKS: but the Notion is intirely groundless. It is so far from having such a Tendency, that it manifestly conduces to dispel the Ignorance of many, who practice Physick, and to furnish them with that Knowledge, which may well raise them above that Ignominious Character."

Lobb's view was clearly an enlightened one; however, I wonder how many doctors could write their papers in Latin today?

Submitted by A P Radford, retired general practitioner, Taunton, Somerset 